\title{
STM capacity for Chinese and English language materials
}

\author{
BOLIN YU, WUTIAN ZHANG, QICHENG JING, and RUIXIANG PENG \\ and \\ GUOJUN ZHANG and HERBERT A. SIMON \\ Carnegie-Mellon University, Pittsburgh, Pennsylvania
}

Institute of Psychology, Chinese Academy of Sciences, Beijing, People's Republic of China

\begin{abstract}
This paper delineates the theoretical implications of a program of research on short-term memory using Chinese characters as stimuli, and compares the findings with studies of short-term memory that use English language materials. The 14 experiments on which it is principally based, carried out in the People's Republic of China and in the U.S.A., are reported in detail in Yu et al. (1984), W. Zhang et al. (1984), and G. Zhang and Simon (this issue). One major theoretical product of this research is an experimentally tested model that reconciles the chunking theory of STM capacity with the articulatory loop theory of Baddeley, making good quantitative predictions of capacity compatible with both. Another result, obtained by using homophones as stimuli, is a demonstration that STM is mainly acoustically encoded, but that there are an additional two or three chunks of visually or semantically encoded short-term memory available. Chunks are shown to play the same role in immediate recall and rote learning in both the Chinese language and English language stimuli; and STM capacity, measured in chunks, is essentially the same for materials in both languages.
\end{abstract}

This paper examines the findings of a series of some 14 experiments on short-term memory carried out with Chinese language stimuli; proposes an STM model to account for the findings; and analyzes the relation of the findings to the literature on short-term memory that relies mainly on experiments with English language materials. The paper discusses the relation of the "chunking" hypothesis (Chase \& Simon, 1973a, 1973b; Miller, 1956; Simon, 1974) of STM capacity to Alan Baddeley's (1981, 1983; Baddeley, Thomson \& Buchanan, 1975; Salame \& Baddeley, 1982; Vallar \& Baddeley, 1982) rehearsal loop hypothesis, and shows how the two can be reconciled. It provides new evidence about the role of acoustic and nonacoustic encodings of STM materials, ${ }^{1}$ and gives several examples of how the decomposability of Chinese characters (into radicals and phonetics) and the existence of numerous homophones of most characters provide means for exploring STM that are not available with English language materials.

The experiments were carried out by three (overlapping) research groups, two at the Institute of Psychology, Chinese Academy of Sciences, Beijing, and one at the Department of Psychology, Carnegie-Mellon University, Pittsburgh. The experiments in Beijing were performed during the Spring of 1983, and the Pittsburgh experiments, during the first 6 months of the same year. Although all

Guojun Zhang's and H. A. Simon's mailing address is: Department of Psychology, Carnegie-Mellon University, Schenley Park, Pittsburgh, PA 15213. of the experiments use the same basic immediate recall paradigm, there are some small differences in method among the three groups. These differences provide opportunities for testing the robustness of the findings. They will be mentioned in the appropriate places.

The written Chinese language provides opportunities to study the structure and capacity of STM under conditions that are quite different from those of alphabetic languages. The basic unit of Chinese writing is the character, or "hanzi," an arrangement of strokes that is ideographic in its origins, and each of which corresponds to a definite spoken syllable. An average Chinese college student is familiar with perhaps 5,000 hanzi; the Xinhua Zidian, a widely used character dictionary, contains about 11,000 hanzi.

Most hanzi are made up of two well-defined substructures or contain radicals, frequently occurring component units made up of definite strokes (see Figure 1a). Compound characters of three or more substructures are relatively few (see Figure 1b).

Hanzi are units both for writing and pronunciation. Some of them correspond to Western one-syllable words, having definite meaning by themselves. But about $70 \%$ to $80 \%$ of Chinese words, called "ci," are of two syllables; that is, they correspond to two characters. There are a few three- and four-character ci. There are also many familiar Chinese idioms, $90 \%$ of which are composed of four characters each.

Most of the ci that are composed of more than one character have a definite meaning that no one could understand, in oral or written Chinese, solely from a 


\section{(a) Radicals}

(b)

Compound Choracters

of three substructures

ory set forth in Miller's (1956) "Magical Number Seven" paper. The second is Baddeley's (1983) theory of an articulatory loop that has to be rehearsed (covertly or overtly) within a limited time interval to retain the information.

Several other prominent theories of short-term memory,

Figure 1. Radicals and compound characters of three substructures.

knowledge of the meanings of the component hanzi. The Changyong Gouci Zidian (a dictionary of high-frequency characters) contains some 4,000 hanzi and more than 90,000 multiple-character words (ci). The Hanying Cidian, a bilingual Chinese-English dictionary, contains some 6,000 hanzi (including one-character ci) and more than 50,000 words (multicharacter ci), the latter number being comparable to the number of entries in Webster's Collegiate Dictionary. ${ }^{2}$

There are several reasons, to which we have already alluded briefly, why experiments on short-term memory with Chinese language materials should be of considerable theoretical interest. First, since Chinese characters are themselves analyzable into components (radicals and phonetics), they provide stimuli that can be manipulated in various ways to determine how they are structured in terms of chunks. For example, artificial characters can be constructed by combining familiar radicals and phonetics in unfamiliar configurations. Second, since only about 1,200 distinct pronounceable syllables are available in the Chinese language to be matched to a much larger number (say, 6,000) of distinct characters, each character has, on average, nearly a half dozen exact homophones. Hence, graphic and phonemic similarity can be manipulated independently and in a precise manner.

Third, theories about chunking have been tested almost exclusively with materials from Western languages. The Chinese language provides valuable stimulus material for testing the generality of these theories and of the parameters of short-term memory that have been estimated from Western materials.

In this summary paper, we wish to review the main scientific questions that were addressed in these experiments, and to bring together the findings of the three studies that bear on these questions. We will discuss: (1) effects on STM capacity of chunking and articulation time; (2) effects on STM capacity of chunk complexity and frequency, and of homophony; (3) STM capacity for character components (radicals); (4) visual (or semantic) STM; (5) procedural differences among the studies; and (6) relation of STM to LTM fixation time.

\section{CHUNKING VERSUS ARTICULATION THEORIES OF CAPACITY}

In the contemporary cognitive literature, a number of proposed explanations can be found for the limited capacity of short-term memory. We will be concerned mainly with two of these. The first is the chunking the-

which are variants or elaborations of the chunking theory, do not need to be distinguished from it for present purposes. One of these is the 1961 EPAM theory of Feigenbaum (Feigenbaum \& Simon, 1984); a second is the spreading activation theory of Anderson (1983) and others; and a third is Estes's (1972) model of short-term memory for serial order.

In its simplest form, the chunking theory predicts that the short-term memory will hold a fixed number of familiar stimulus units, the number being independent of the nature of the units so long as they are all familiar chunks. In contrast, the articulatory loop theory predicts that short-term memory will hold a fixed number of syllables (more precisely, that it will hold the number of syllables that can be pronounced in a fixed amount of time).

We can test these theories by measuring STM capacity with stimuli that vary both in numbers of chunks and numbers of syllables per chunk. The chunking theory predicts that the same number of chunks will be retained, regardless of the number of syllables per chunk. The articulatory theory predicts that the same number of syllables will be retained, regardless of the number of familiar chunks of which these syllables are composed.

The data from these experiments with Chinese language stimuli, as well as a review of previous experiments with English language stimuli, show that neither theory tells the whole truth. The findings, in fact, show that both chunks and syllables affect retention.

In their Experiments 1, 2, and 4, Yu, Jing, and Sima (1984) compared memory spans for one-character nouns, two-character nouns, and four-character idioms, using both simultaneous and serial modes of presentation, and characters of low and high frequency. In all cases, memory span measured in chunks decreased significantly (t test, $\mathrm{p}<.01$ ) with number of syllables per chunk, whereas the span measured in syllables increased. W. Zhang, Peng, and Sima (1984), in their Experiment 1, obtained a similar result (also significant at the .01 level), using a serial mode of presentation. In their Experiment 1, G. Zhang and Simon (1985) found the same effect ( $p<$ .001 ) in comparing single characters with two-character words and, in their Experiment 6, in comparing single characters, two-character words, and four-character idioms (all $\mathrm{ps}<.01$ by $\mathrm{t}$ test). These findings are wholly consistent with those of previous experiments using English language materials; memory span decreases, as measured in chunks, with an increase in syllables per chunk, but the span measured in syllables increases.

In G. Zhang and Simon (1985), a theory is proposed that explains these empirical findings on the basis of a mechanism that incorporates both chunking and syllable articulation. The new theory postulates an articulatory 
loop of fixed duration, as Baddeley (1981) does, but postulates that time is required not only to articulate syllables. but also to retrieve each new chunk for the articulatory mechanism.

The new model has three parameters, $T$, $a$, and $b$, corresponding to the duration of the articulatory loop, the unit time for retrieving a chunk, and the unit time for articulating a syllable, respectively. However, since only the ratios of $T$ to the other two parameters can be estimated from the data, there are two degrees of freedom in the fit. The parameter estimates obtained by fitting data from six different experiments, four with Chinese language stimuli and two with English, are shown in Table 1. All estimates assume $T=2.0 \mathrm{sec}$, a value proposed by Baddeley et al. (1975) from data on reading speeds.

The estimates of the chunk retrieval time, a, are all in good agreement with each other, the smallest being only $24 \%$ less than the largest. The values cluster in the neighborhood of $300 \mathrm{msec}$. There is greater variation among the estimates of the syllable articulation times, the longest being nearly three times the shortest. The English language estimates ( 53.2 and $66.9 \mathrm{msec}$ ) fall within the range of the Chinese language estimates ( 48.8 to $130.6 \mathrm{msec}$ ).

These numbers may be compared with the $160 \mathrm{msec} /$ letter that is commonly reported as the rate for covert rehearsal of the English alphabet, and Baddeley et al's (1975) data on reading rates that give an estimate of $83.7 \mathrm{msec} / \mathrm{syllable}$. Assuming a mean of $300 \mathrm{sec}$ for chunk retrieval time and $80 \mathrm{msec}$ for syllable articulation time, the alphabet, in seven chunks (ABCD EFG HIJK LMNOP QRST UVW XYZ), would be rehearsed at $(300 \times 7+80 \times 26) / 26=161 \mathrm{msec}$ per syllable, in good agreement with the observed times.

Differences in experimental method may provide part of the explanation for the observed variations in articulation time. In the Chinese data in Table 1, the two largest estimates (130.6 and $120.1 \mathrm{msec}$ ) come from experiments in which all stimuli in a sequence were presented simultaneously. The lowest estimate $(48.8 \mathrm{msec})$ comes from an experiment in which presentation was sequential. The intermediate estimate $(86.4 \mathrm{msec})$ is from experiments in which presentation was sequential but cumulative (see $\mathrm{Yu}$ et al., 1984, for details).

We cannot regard any of these interpretations of the variation in $b$ as conclusive. Meanwhile, the theory com-

Table 1

Parameter Estimates For $a$ and $b$ (in Milliseconds) in $C=2000 /[a+b(S-1)]$

\begin{tabular}{lcc}
\hline Source & Chunk Retrieval Time & Syllable Time \\
\hline Yu* & 373.6 & 130.6 \\
Yu ${ }^{*}$ & 286.2 & 86.4 \\
W. Zhang & 297.6 & 48.8 \\
G. Zhang & 309.1 & 120.1 \\
Simon 1974 & 281.2 & 53.2 \\
Baddeley & 285.6 & 66.9 \\
\hline
\end{tabular}

* Simultaneous presentation method. †Serial-simultaneous presentation method. bining chunking and articulatory mechanisms fits the data from each of our experiments with much better than orderof-magnitude agreement in the values of the parameters. And, equally remarkable, the Chinese language findings are in good agreement with those from English language materials. The theory can therefore claim considerable power and generality.

\section{CHUNK COMPLEXITY, HOMOPHONY, FREQUENCY}

In their Experiment 1, Yu et al. (1984) found a significant $(\mathrm{p}<.01$ by $t$ test) relation between memory span and frequency of one-syllable nouns (characters). In their Experiment 2, there was no significant effect of frequency upon the spans of two-character words or four-character idioms. In their Experiments 1, 2, and 3, W. Zhang et al. (1984) also compared spans for low-and high-frequency one-, two-, and three-character stimuli, using different presentation (visual or auditory) and response (written or oral) modes, finding no significant differences.

As the authors point out, in the materials of these experiments, frequency is confounded with complexity (number of strokes required to write each character). Additional experiments will be required to unconfound these two variables, but meanwhile there are other complications that should be mentioned as well.

As we have already explained briefly, the individual characters used to write the Chinese language do not correspond closely to English words. In Chinese, each character is pronounced as a single syllable ( $\mathrm{CV}$ or $\mathrm{CVC}$ ). The basic lexical entry in Chinese, corresponding to an English word, may consist of one (e.g., "chi" - to eat), two (e.g., "wenti"-problem), or more (e.g., "tushuguan"--library) characters (and syllables), two being the modal number. The frequency data we used to classify characters refer to the frequencies of individual characters, independently of whether or not they were embedded in words. At present, only approximate data are available on the frequencies of occurrence of Chinese words.

Since each character is associated with a single-syllable pronunciation, and taking account of the fact that each vowel may be pronounced in any one of four tones, there are a little more than 1,000 distinguishable syllables in the Chinese language that must be allocated to the 5,0007,000 characters in general use. This means that each character has, on average, about six homophonescharacters that are visually and semantically quite distinct, but that are pronounced in exactly the same way. Chinese words, being composed typically of several characters, do not as frequently have homophones, although there is probably a greater occurrence of homophones in Chinese than in English, even at the word level.

Now, in the data that relate STM capacity to character frequency and complexity, not only are frequency and complexity confounded, but both are confounded with homophony as well. To see why this is so, consider an example. There are, in standard Chinese dictionaries, 
about 10 characters, all visually different, that are pronounced "gong"' with high tone. Suppose that one of these is presented visually in an immediate-recall task. If the subject encodes the information in the stimulus only acoustically, then the ability to discriminate among the homophones will be lost. When attempting to make a written response, the subject will have to choose (arbitrarily) one of the homophones. The simplest hypothesis is that the one with highest frequency of use will usually be chosen, regardless of which one was presented as the original stimulus. Hence, if this frequently used homophone is the correct response, there will be few homophone errors, whereas if it is not, the response will usually be incorrect. This mechanism can account for the very large difference in STM span between high-frequency and low-frequency single-character words found in Yu et al.'s (1984) Experiment 1 and the sizable, though statistically insignificant, differences in the same direction for singlecharacter stimuli in Zhang et al.'s (1984) Experiments 1 and 3.

Zhang and Simon's (1985) Experiment 4 provides additional strong supporting evidence for this explanation of the effect of frequency. This experiment employed three stimulus lists: the first consisted of characters that had no homophones of higher frequency; the second, of characters that had a single homophone of higher frequency; and the third, of characters having four or more homophones of higher frequency. Significant differences in STM span were found among these lists comparable to the differences in lists of high- and low-frequency characters found by Yu et al. (1984). Moreover, when homophonic responses were counted as correct, the differences became much smaller, and lost, or nearly lost, their statistical significance.

Further support for this explanation comes from $\mathrm{Yu}$ et al.'s (1984) Experiment 2. Low-frequency twocharacter words and four-character idioms were compared with high-frequency two-character words and fourcharacter idioms, respectively. (The indirect measures of frequency used in constructing these lists are described in that paper.) Two-character words seldom have homophones, and four-character idioms almost never do. Correspondingly, no differences in memory span were found for the low-frequency and high-frequency lists. We find, therefore, very strong support for the hypothesis that a major part of the effect of frequency (and perhaps of complexity) is mediated by the presence or absence of homophony.

\section{CAPACITY FOR RADICALS AND OTHER COMPONENTS}

Chinese characters cannot only be combined to form words; they can also be decomposed into component parts that are themselves often familiar chunks. There is great variation in the ways in which characters are formed, but one common pattern is a character that consists of two parts: a radical, which often conveys some semantic in- formation about the character, and a phonetic, which often conveys some information about its pronunciation. Neither piece of information is either definitive or reliable, but each has heuristic or mnemonic value in identifying the character or retaining it in memory.

Some radicals and phonetics can also serve as independent characters, but many cannot, or are severely distorted when so used, as compared with the free-standing form. Most radicals are encountered frequently by Chinese readers (there are only about 200 of them in all), but usually in the context of characters containing other parts as well. Dictionaries are indexed by radicals, so that the latter are used often to search for characters. Not all radicals have well-known names, and their names are usually multisyllabic. We may ask, then, whether radicals will behave like chunks in immediate recall, and whether they will give memory spans similar to those of independent characters.

Yu et al.'s (1984) Experiment 3 and G. Zhang and Simon's (1985) Experiment 1 show that the memory span for radicals is substantially (and significantly) smaller than the span for characters. In both experiments, however, there was a considerable difference in the measured spans, which reflected a difference in the nature of the radicals used as stimuli. In the Zhang-Simon experiment, where the mean span was only 2.71 , radicals that were relatively uncommon and had no simple names were deliberately selected as stimuli. In the Yu et al. experiment, where the mean span was 4.55 radicals, the radicals were relatively common ones that do have well-known names, although not monosyllabic ones. Hence, it appears plausible that the ability to name the stimulus, and to name it succinctly, was a major factor in determining memory span. However, comparison by Yu et al. of the mean span for radicals of 4.55 in their Experiment 3 with the mean span for characters of medium frequency, 5.68, in their Experiment 1, shows the latter to be significantly larger than the former ( $p<.01$ by $t$ test). Therefore, the ability to name the stimuli does not account for the entire difference in span.

In their Experiment 3, Yu et al. (1984) compared memory spans for (named) radicals, for pairs of unrelated characters, for pseudocharacters (noncharacters obeying the rules of syntax for the construction of characters from components), noncharacters (constructed from components, but in violation of the rules of syntax), and pairs of radicals. Each of the stimuli, other than the individual radicals, had two components. Memory spans decreased from the first category listed above to the last, but the differences in span were not statistically significant.

The spans obtained with these stimulus materials are consistent with the idea that the components of these artificial constructs, and not the entire constructs, behave as chunks. The mean spans for the stimuli were very small2.70 for pairs of characters, 2.40 for stimuli constructed according to the syntax of Chinese characters (pseudocharacters), 2.25 for those that violated the syntax (noncharacters), and 1.80 for pairs of radicals. If we multi- 
ply these numbers by two, on the assumption that each of the two components of the artificial characters were familiar chunks, we obtain spans of 5.40, 4.80, 4.50, and 3.60 , which are not far from the span (4.55) for isolated radicals.

\section{VISUAL OR SEMANTIC STM}

From the data on "nameless" radicals in G. Zhang and Simon's (1985) Experiment 1, we have seen that the memory span for nonpronounceable materials may be as low as two or three units. And when the stimuli are all perfect homophones, as in G. Zhang and Simon's Experiment 2, the span is again about this size. These findings provide strong evidence for a small nonacoustical shortterm memory. None of the experiments contain evidence that would allow us to decide whether this memory is visual or semantic-whether it retains information about the pictorial features of the characters or information about their meaning. Nor do we have evidence as to whether the acoustical and nonacoustical memories can be used to augment each other or can operate wholly independently.

\section{EFFECTS OF PROCEDURAL DIFFERENCES}

\section{Sequential and Simultaneous Presentation}

The immediate recall experiments in the three experimental papers generally employed the same paradigm, with one important difference. In G. Zhang and Simon's (1985) experiments, each stimulus sequence was presented on a single card; hence, the individual components of the sequence were available to the subjects simultaneously. The same procedure was followed in Yu et al.'s (1984) first three experiments. In W. Zhang et al.'s experiments, however, each item of each stimulus sequence was presented independently of those that preceded and followed it.

Since W. Zhang et al.'s (1984) Experiment 1 showed less effect of character frequency upon memory span than did Yu et al.'s (1984) Experiment 1, an additional experiment (Experiment 4) was run by Yu et al. to test the interaction of the effects of frequency and method of presentation. In the new experiment, a serial method of presentation was adopted, but each syllable remained on the screen until all items had been presented. The experimenters called this the "serial-cumulative" method.

Comparison of the results for the three methods showed that memory span was larger and less dependent on stimulus complexity (number of strokes) for the serial method than for the serial-cumulative method, and for the serialcumulative method than for the simultaneous methods. These results are discussed further in Yu et al. (1984), where it is suggested that the differences in method of presentation of the stimuli may cause differences in the way in which subjects allocate their attention to different stimuli.

\section{Visual and Acoustical Presentation}

W. Zhang et al. (1984), in their Experiment 3, compared oral and visual presentation of characters, words, and idioms. Since, with oral presentation, homophones cannot be discriminated, homophonic responses were counted as correct. In all conditions, there was slightly better memory for visually presented material than for orally presented material, but the differences were not statistically significant.

\section{LONG-TERM MEMORY FIXATION}

It has been found (Simon, 1974), that, with English language materials, there is a close correlation between chunk size as measured by short-term memory capacity and chunk size as measured by time required for fixation of stimuli in long-term memory when they are learned by a paired-associate or serial-anticipation paradigm. That is to say, the time required to memorize a sequence of stimuli is proportional to the number of chunks (determined by an immediate-recall experiment) in the sequence. The factor of proportionality is about $8 \mathrm{sec}$ per chunk, with learning to the criterion of one correct repetition. Thus, two converging procedures, measuring immediate recall spans and rote memory speeds, respectively, provide consistent definitions of chunks.

As a further comparison between English language and Chinese language materials, W. Zhang et al. (1984) compared the times required to learn one-character and twocharacter words, respectively, by the serial anticipation method, with both visual and oral presentation of the stimuli. They found only small differences in learning times between one-character and two-character words. Learning times were in the range of 8 to $11 \mathrm{sec} / \mathrm{chunk}$. Hence, the results for English language materials appear, here also, to carry over to the Chinese materials. There is good correspondence between the chunks defined by the two paradigms, and good agreement in learning times between the Chinese language and English language experiments.

\section{CONCLUSION}

In this paper, we have compared short-term memory span for Chinese characters, components of characters, and words with memory spans for English words. A model based on Baddeley's articulatory loop hypothesis, but allowing time (about $300 \mathrm{msec}$ ) for retrieval of chunks as well as articulatory time (about $80 \mathrm{msec} / \mathrm{syllable),} \mathrm{fits}$ the data very well, and the parameters estimated from Chinese language data fall in the same range as the parameters estimated from English language data.

In addition, we have used the presence of numerous homophones among Chinese characters as a means of separating the acoustic and nonacoustic components of short-term memory. We find that the short-term memory span for chunks that can be discriminated and encoded 
acoustically is in the neighborhood of six or seven, whereas the span for homophones that can be discriminated only visually or semantically but not acoustically is only two or three.

Components of Chinese characters that do not have readily pronounceable names (e.g., radicals) have relatively short spans, as do pseudocharacters that are synthesized from familiar components. These and other effects mentioned in the body of the paper are consistent with the model, and with the general hypothesis that chunks are familiar units of any kind that can be named and retained in short-term memory in an acoustic encoding. No effects were detected that are peculiar to ideographic or logographic languages in contrast to alphabetic languages.

\section{REFERENCES}

ANDERson, J. R. (1983). The architecture of cognition. Cambridge, MA: Harvard University Press.

BADDELEY, A. D. (1981). The concept of working memory: A view of its current state and probable future development. Cognition, 10, 17-23.

BADDEley, A. D. (1983). Working memory. Philosophical Transactions of the Royal Society, London, B302, 311-324.

Baddeley, A. D., Thomson, N., \& Buchanan, M. (1975). Word length and structure of short-term memory. Journal of Verbal Learning and Verbal Behavior, 14, 575-589.

Chase, W. G., \&imon, H. A. (1973a). The mind's eye in chess. In W. G. Chase (Ed.), Visual information processing. New York: Academic Press.

Chase, W. G., \& Simon, H. A. (1973b). Perception in chess. Cognitive Psychology, 4, 55-81.

ESTES, W. K. (1972). An associative basis for coding and organization in memory. In A. W. Melton \& E. Martin (Eds.), Coding processes in human memory. Washington: Wiley.

Feigenbaum, E. A., \& Simon, H. A. (1984). EPAM-like models of recognition and learning. Cognitive Science, $8,305-336$.
MiLler, G. A. (1956). The magical number seven plus or minus two: Some limits on our capacity for processing information. Psychological Review, 63, 81-87.

Salame, P., \& Baddeley, A. D. (1982). Disruption of short-term memory by unattended speech: Implications for the structure of working memory. Journal of Verbal Learning and Verbal Behavior, 21, 150-164.

Simon, H. A. (1974). How big is a chunk? Science, 183, 482-488.

VALLAR, G., \& BADDELEY, A. D. (1982). Short-term forgetting and the articulatory loop. Quarterly Journal of Experimental Psychology, 34A, 53-60.

YU, B., JiNG, Q., \& SimA, H. (1984). STM capacity for Chinese words and phrases under simultaneous presentations. In National Academy of Sciences, Chinese Academy of Sciences, Issues in Cognition: Proceedings of a Joint Conference in Psychology. Washington: National Academy of Sciences \& American Psychological Association.

ZhANG, W., \& Simon, H. A. (1985). STM capacity for Chinese words and idioms: Chunking and acoustical loop hypotheses. Memory \& Cognition, 13, 193-201

Zhang, W., Peng, R., \& Sima, H. (1984). STM capacity for Chinese words and idioms with visual and auditory presentations. In National Academy of Sciences, Chinese Academy of Sciences, Issues in Cognition: Proceedings of a Joint Conference in Psychology. Washington: National Academy of Sciences \& American Psychological Association.

\section{NOTES}

1. Throughout this paper, "acoustic" will be used as a generic term to refer to encodings of auditory stimuli, or to stimuli presumed to be encoded in some auditory or phonemic or articulatory code. We will not have occasion to make finer discriminations of encodings that relate to signals in the acoustic mode.

2. A character dictionary, containing hanzi, is called "zidian"; a word dictionary that contains one-character and multicharacter ci is called "cidian."

(Manuscript received February 3, 1984; revision accepted for publication March 15, 1985.) 